\title{
GROUND-STATE ENERGY AND COMPRESSIBILITY OF A DISORDERED TWO-DIMENSIONAL ELECTRON GAS
}

\author{
B. TANATAR* and A. L. SUBAŞI \\ Department of Physics, Bilkent University, \\ Bilkent, Ankara, 06800, Turkey \\ *E-mail: tanatar@fen.bilkent.edu.tr \\ K. ESFARJANI \\ Department of Physics, Sharif University of Technology, \\ Tehran, Iran \\ Department of Physics, University of California, Santa Cruz, CA 95064 \\ S. M. FAZELI \\ Department of Physics, Sharif University of Technology, \\ Tehran, Iran
}

\begin{abstract}
Two-dimensional (2D) electron systems in the presence of disorder are of interest in connection with the observed metal-insulator transition in such systems. We use density functional theory in its local-spin density approximation (LSDA) to calculate the ground-state energy of a $2 \mathrm{D}$ electron system in the presence of remote charged impurities which up on averaging provides disorder. The inverse compressibility calculated from the ground-state energy exhibits a minimum at a critical density controlled by the disorder strength. Our findings are in agreement with experimental results.
\end{abstract}

Keywords: 2D electron gas; disorder effects; compressibility.

\section{Introduction}

There has been an immense amount of experimental and theoretical activity in recent years to understand the ground state properties of two-dimensional (2D) electron systems. ${ }^{1,2}$ Advances in fabrication techniques have made it possible to probe various quantities of interest in high quality and very low density samples. Most notably, the observation of a metal-insulator transition ${ }^{1,2}$ in these systems provides a major motivation to study the various physical properties. Most experiments perform transport measurements obtaining resistivity or conductivity as a function of temperature at varying electron density to deduce the metallic or insulating phases. ${ }^{3,4,5,6}$ In contrast, Ilani et al. ${ }^{7}$ used the capacitance technique, a thermodynamic measurement, to measure the compressibility finding that it becomes spatially inhomogeneous as the density is lowered. Similar observations were also made by Dultz and Jiang ${ }^{8}$ on a $2 \mathrm{D}$ hole system who noted that the inverse 
compressibility is minimum at the same density where the metal-insulator transition occurs. Recent compressibility and resistivity experiments by Allison et al. ${ }^{9}$ on the same samples indicate that inverse compressibility minimum is not related to metal-insulator transition.

Most of our understanding of metal-insulator transition in 2D electron systems is based on various transport measurements. For instance, experiments at in-plane magnetic field concentrated on the spin susceptibility, Lande $g$-factor, and effective mass of the 2D electron systems present in Si-MOSFETS and GaAs quantum-well structures $\mathrm{s}^{3,4,5,6,10,11}$. In particular, Shashkin et al. ${ }^{12,13}$ reported a sharp increase of the effective mass near the critical density at which the system starts to show deviations from the metallic behavior. On the other hand, Pudalov et al. ${ }^{3}$ have found only moderate enhancement of the spin susceptibility and effective mass in their samples. Thermodynamic measurements of magnetization of a dilute $2 \mathrm{D}$ electron system were reported by Prus et al. ${ }^{10}$ and Shashkin et al. ${ }^{11}$ Both experiments found large enhancement of the spin susceptibility $\chi_{s}$ over its Pauli value. Whereas the measurements of Prus et al. ${ }^{10}$ found no indication toward a ferromagnetic instability, Shashkin et al. ${ }^{11}$ observed diverging behavior in $\chi_{s}$ at a critical density coinciding with the metal-insulator transition density obtained from transport measurements.

As indicated above, there are very few thermodynamic measurements at densities around the metal-insulator transition. As recent theoretical work by Punnoose and Finkelstein ${ }^{14}$ shows the observed behavior in $2 \mathrm{D}$ systems suggests an interaction driven mechanism with disorder playing an important role. On the theoretical side, calculations of compressibility for a $2 \mathrm{D}$ system of electrons in the presence of disorder predict the observed behavior of upturn and divergence ${ }^{15,16}$. Shi and $\mathrm{Xie}^{17}$ performed density functional calculations based on the (unpolarized) local density approximation developed by Tanatar and Ceperley ${ }^{18}$ within the Thomas-Fermi (TF) theory, and found similar results for the compressibility. They also identified the MIT point with the percolation transition point in this system. This was further developed by Das Sarma et al. ${ }^{19}$ who measured the critical exponent for the conductivity and found it in agreement with that proposed by percolation theory. On the other hand, Fogler ${ }^{20}$ argued against percolation transition by calculating the critical density to be much smaller.

In this work, we investigate the spatial distribution of carrier density and the compressibility of a $2 \mathrm{D}$ electron system using the local spin-density approximation (LSDA) both at the TF and Kohn-Sham (KS) levels. The correlation energy functional we use is the one constructed by Attaccalite et al. ${ }^{21}$ from the very recent quantum Monte Carlo (QMC) calculations for correlation energy appropriate for uniform systems. An important feature of these simulations is that a transition to a ferromagnetic phase at low densities is built in the functional. A disorder potential due to remote impurities is included to make the calculation realistic. The density distribution of the system shows high and low density regions as reported earlier by Shi and Xie. ${ }^{17}$ Our density functional theory calculations clearly show that the observed minimum behavior in inverse compressibility is the result of electron-impurity 
interaction. The critical density at which the minimum occurs is controlled by the disorder strength.

In the rest of this paper, we first outline the density functional theory as applied to $2 \mathrm{D}$ electron systems with impurity interaction, and introduce our computational method. We then present our results for the ground-state energy and compressibility for $2 \mathrm{D}$ electrons in the presence of disorder and discuss our findings in the light of recent experiments.

\section{Theory}

We consider a $2 \mathrm{D}$ electron system interacting via the long range Coulomb interaction whose Fourier transform is $V_{q}=2 \pi e^{2} /(\epsilon q)$ in which $\epsilon$ is the background dielectric constant. The system is characterized by the dimensionless interaction strength $r_{s}=1 /\left(\pi n a_{B}^{*}\right)^{1 / 2}$, where $n$ is the $2 \mathrm{D}$ electron density and $a_{B}^{*}=\hbar^{2} \epsilon /\left(m^{*} e^{2}\right)$ is the effective Bohr radius defined in terms of the band mass $m^{*}$ of electrons in the semiconductor structure and dielectric constant $\epsilon$ of the material.

Within the spin-density functional theory the total energy of an $N$-electron interacting system in a local external potential $V_{\text {ext }}(\mathbf{r})$ is a unique functional of spin densities $n_{\uparrow}(\mathbf{r})$ and $n_{\downarrow}(\mathbf{r})$. The total energy functional can be expressed as

$$
\begin{array}{r}
E_{\text {Total }}\left[n_{\uparrow}, n_{\downarrow}\right]=E_{T}\left[n_{\uparrow}, n_{\downarrow}\right]+E_{H}\left[n_{\uparrow}, n_{\downarrow}\right] \\
+E_{x}\left[n_{\uparrow}, n_{\downarrow}\right]+E_{c}\left[n_{\uparrow}, n_{\downarrow}\right]+E_{\text {ext }}\left[n_{\uparrow}, n_{\downarrow}\right]
\end{array}
$$

We approximate the kinetic energy functional by the Thomas-Fermi-Weizäcker (TFW) form given by

$$
E_{T}\left[n_{\uparrow}, n_{\downarrow}\right]=\sum_{\sigma} \int d \mathbf{r}\left[\pi n_{\sigma}^{2}(\mathbf{r})+A \frac{\left|\nabla n_{\sigma}(\mathbf{r})\right|^{2}}{n_{\sigma}(\mathbf{r})}\right]
$$

with $A=1 / 8^{22}$. The direct Coulomb energy is given by

$$
E_{H}\left[n_{\uparrow}, n_{\downarrow}\right]=\frac{e^{2}}{2 \epsilon} \int d^{2} \mathbf{r} d^{2} \mathbf{r}^{\prime} \frac{n(\mathbf{r}) n\left(\mathbf{r}^{\prime}\right)}{\left|\mathbf{r}-\mathbf{r}^{\prime}\right|}
$$

where $n(\mathbf{r})=n_{\uparrow}(\mathbf{r})+n_{\downarrow}(\mathbf{r})$ is the total density.

The exchange and correlation energy functionals within the local spin-density approximation are written as

$$
E_{x, c}\left[n_{\uparrow}, n_{\downarrow}\right]=\int d^{2} \mathbf{r} \epsilon_{x, c}\left(n_{\uparrow}(\mathbf{r}), n_{\downarrow}(\mathbf{r})\right)\left[n_{\uparrow}(\mathbf{r})+n_{\downarrow}(\mathbf{r})\right] .
$$

Here $\epsilon_{x}$ is the exchange energy of the 2D homogeneous electron electron gas. For the correlation energy $\epsilon_{c}$ we use the recent QMC result of Attaccalite et al. ${ }^{21}$ again for the $2 \mathrm{D}$ homogeneous electron gas which has been parametrized as a function of density and spin polarization. 
$r_{s}=9.0$
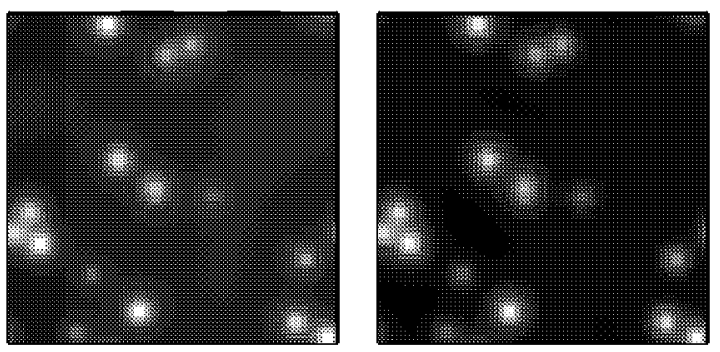

$N_{i}=8, d=3$ a.u.

$r_{s}=6.0$

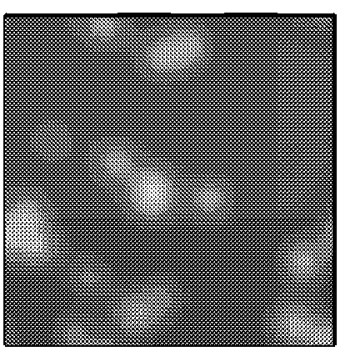

$r_{s}=12.0$

$r_{s}=9.0$

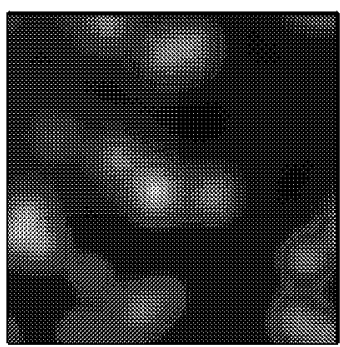

$N_{i}=4, d=1$ a.u.

$r_{s}=15.0$

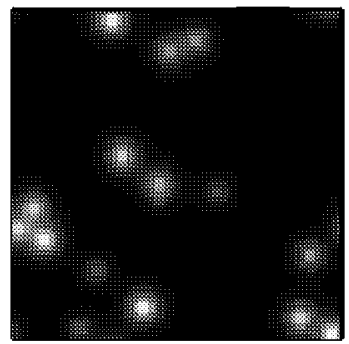

30

25

20

15

$r_{s}=12.0$

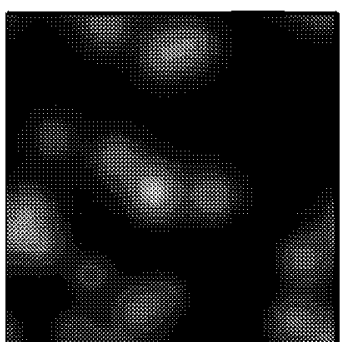

Fig. 1. Density distributions of a $2 \mathrm{D}$ electron system in a model disorder potential for several values of average electron density characterized by $r_{s}$. The numbers on the right scale are in units of $10^{-3}$ a.u. We choose the impurity parameters as upper panel: $N_{i}=4$ and $d=1 \mathrm{a}$.u., lower panel: $N_{i}=8$ and $d=3$ a.u. Ten realizations of the impurity potential are averaged. (colour online)

The disorder studied in this work comes from a random distribution of charged impurities (with charge $Z e$ ) at a setback distance $d$ from the electron layer. The energy functional due to the external potential is

$$
E_{\text {ext }}[n]=\int d^{2} \mathrm{r} V_{\text {ext }}(\mathbf{r})[n(\mathbf{r})]
$$

where the external potential, due to remote impurities, located at $\mathbf{r}_{\mathbf{i}}$ at a distance $d$ from the plane of $2 \mathrm{D}$ electrons is given by

$$
V_{\text {ext }}(\mathbf{r})=-\sum_{i} \frac{Z e^{2} / \epsilon}{\left[\left(\mathbf{r}-\mathbf{r}_{\mathbf{i}}\right)^{2}+d^{2}\right]^{1 / 2}} .
$$

\section{Method}

The spin-densities $n_{\uparrow}(\mathbf{r})$ and $n_{\downarrow}(\mathbf{r})$ that extremize the total energy functional can be obtained by annealing from a Monte Carlo (MC) simulation. A sufficiently high 


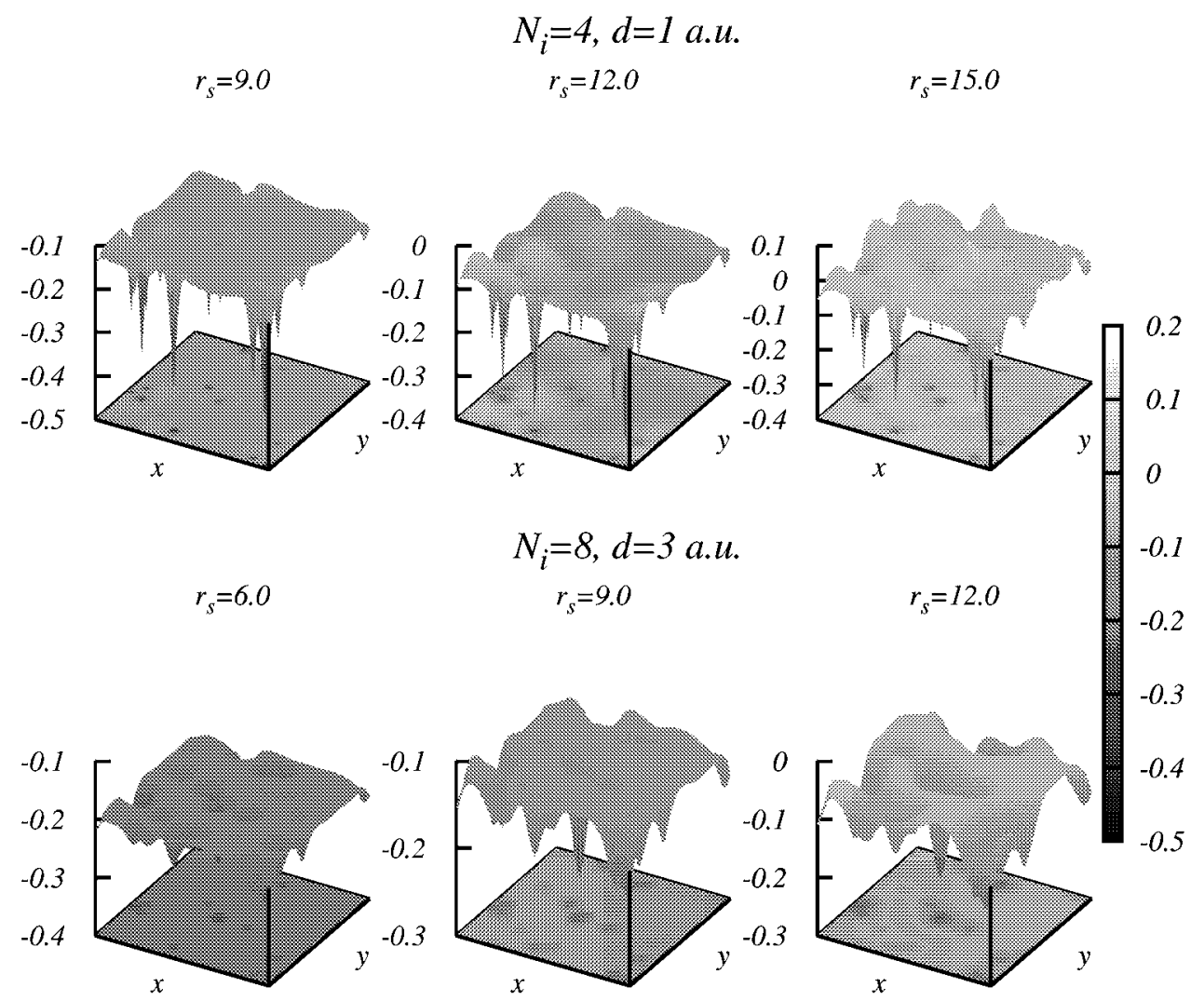

Fig. 2. The effective potential at different disorder strengths and average electron densities characterized b y $r_{s}$. We choose the impurity parameters as upper panel: $N_{i}=4$ and $d=1$ a.u., lower panel: $N_{i}=8$ and $d=3$ a.u. Ten realizations of the impurity potential are averaged.

temperature is first chosen and a Metropolis Monte Carlo run is performed long enough to reach thermodynamic equilibrium. Then the temperature is reduced and the run is repeated. This is continued until the final temperature is sufficiently low so that very little energy fluctuations occur during the last run. This simulation is done in order to reach the global minimum of the energy landscape. Once one is near the bottom of the valley, eventually a steepest-descent algorithm is applied to reach the minimum energy structure faster. The areal integral of the energy functional is approximated by a discrete sum: basically the density and potentials are discretized on a 64 by 64 mesh whose size is one effective Bohr radius being equal to $100 \AA$ in GaAs samples. The long-range Coulomb potential is calculated using the Ewald sum method. In minimizing the total energy functional, if needed, we have considered several different realizations for the impurity atoms and have taken the statistical average.

To make sure of the validity of our analysis, the results from the TFW kinetic energy functional were also compared to the solution of the Kohn-Sham equations 


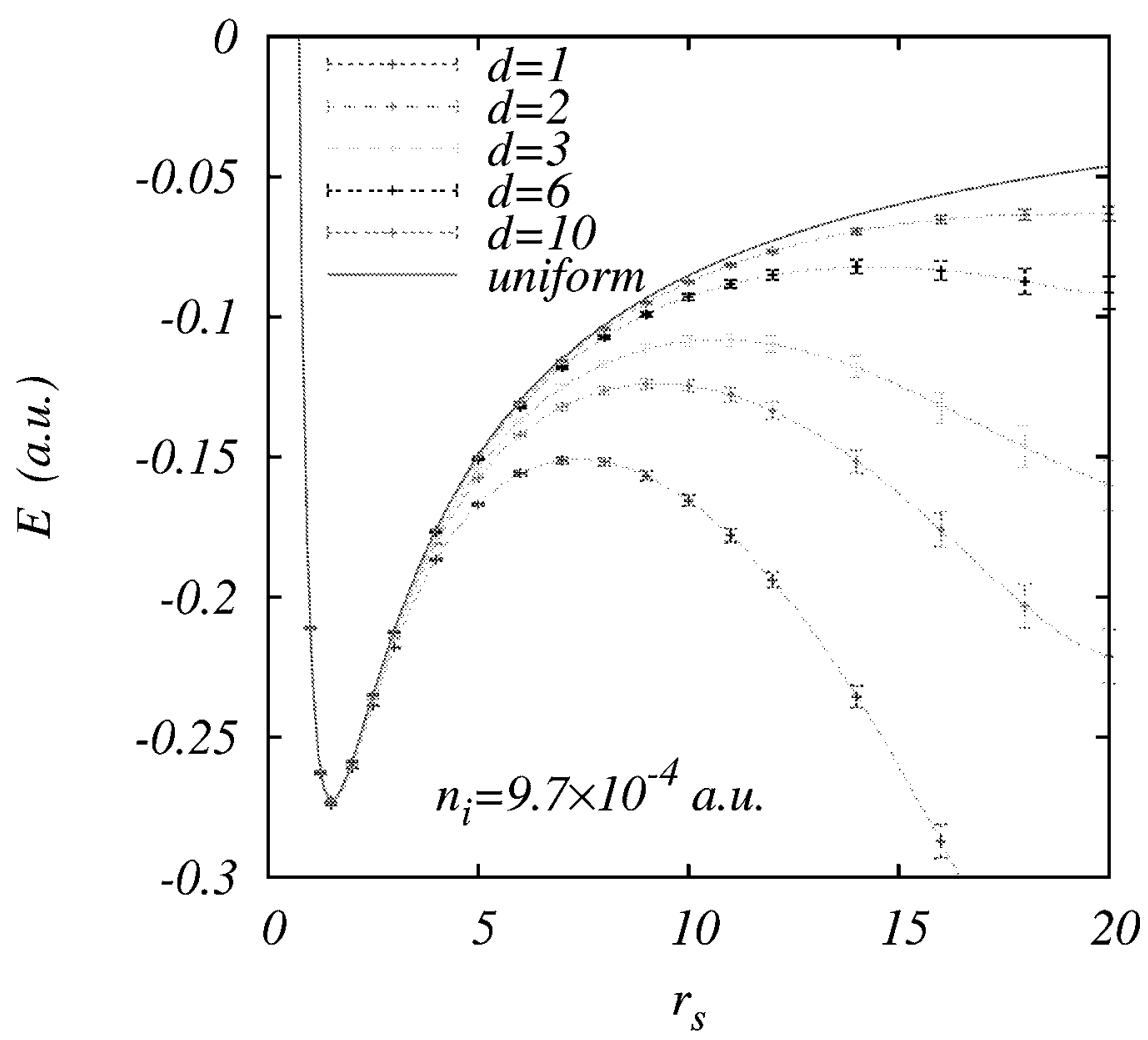

Fig. 3. Ground state energy (per particle) of a 2D electron system in the presence of charged impurities of concentration $n_{i}=0.97 \times 10^{-3}$ a.u. Distance $d$ of the impurities are given in the legend. Homogeneous system result (solid line) is the QMC calculation of Attaccalite et al. (Ref. 21)

where the kinetic energy is calculated exactly. Densities in the high effective potential regions were found to agree to within a few percent. Low potential regions showed good agreement between the densities as well as the effective exchangecorrelation potential. The Kohn-Sham equations follow from the minimization of the total energy functional under the constraint of fixed total number of electrons. The effective single-particle Schrodinger equation is

$$
\left[-\nabla^{2}+V_{\text {eff }}(n, \mathbf{r})\right] \psi_{i s}(\mathrm{r})=\epsilon_{i s} \psi_{i s}(\mathbf{r})
$$

where

$$
V_{\text {eff }}(n, \mathbf{r})=V_{d}(\mathbf{r})+\int d \mathbf{r}^{\prime} \frac{e^{2}}{\left|\mathbf{r}-\mathbf{r}^{\prime}\right|}+\frac{\delta}{\delta n}\left[\epsilon_{x}(n) n+\epsilon_{c}(n) n\right]
$$

is the effective potential self-consistently determined by the density distribution $n(\mathbf{r})=\sum_{i s}\left|\psi_{i s}(\mathbf{r})\right|^{2}$ by summing over the occupied energy levels and the spin index $s$. 


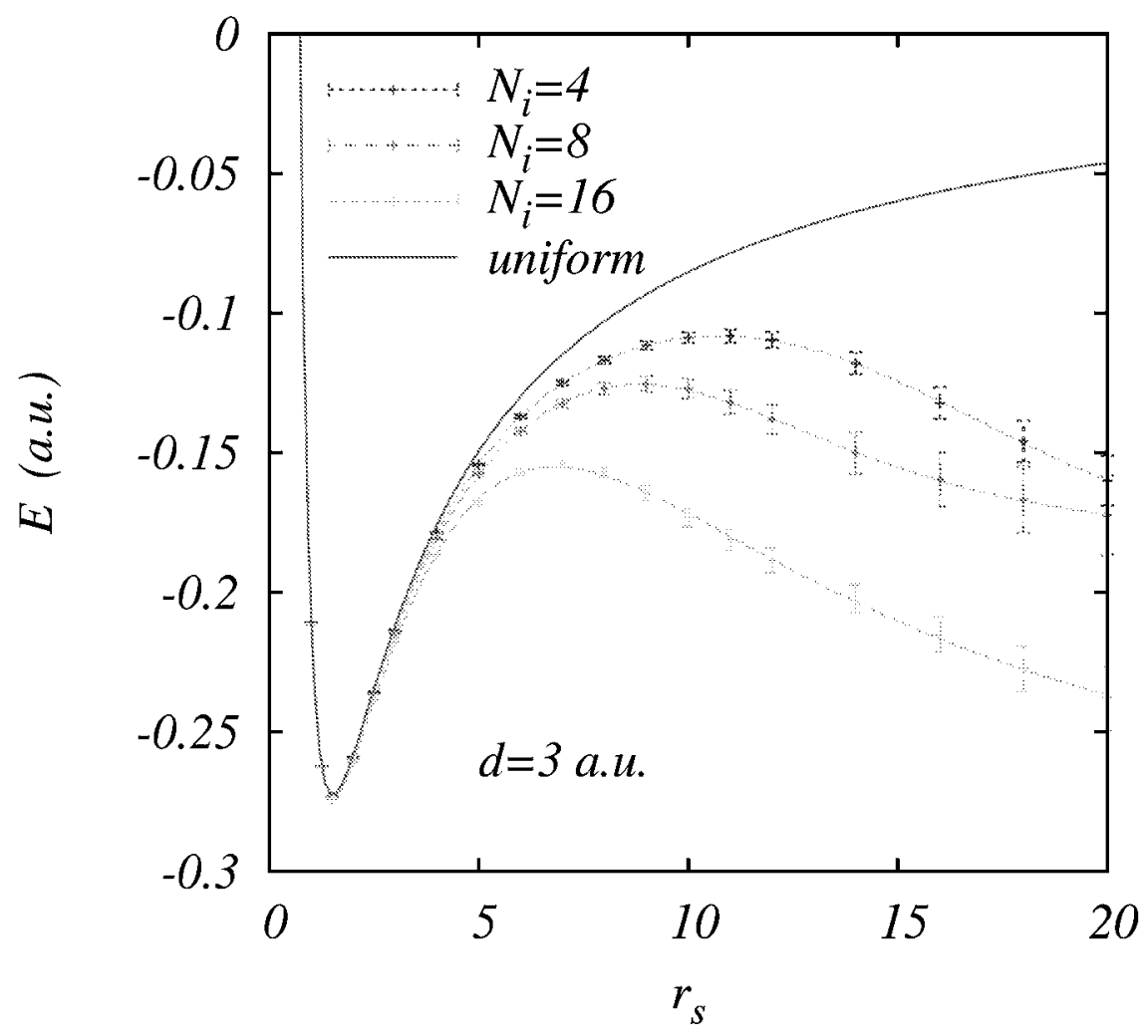

Fig. 4. Ground state energy (per particle) of a 2D electron system in the presence of charged impurities at a setback distance $d=3$ a.u. Number of impurities are given in the legend. Homogeneous system result (solid line) is the QMC calculation of Attaccalite et al. (Ref. 21)

We also remark that a similar Hartree-Fock calculation was recently performed on a Mott insulator in the half-filled limit $^{23}$ where the ground state is antiferromagnetic. We believe that the exchange-correlation potential we are using is more realistic, and contains the correct physics, namely that at very low densities, before the Wigner crystallization, the system becomes ferromagnetic.

\section{Results and Discussion}

We have calculated the ground-state energy and corresponding density distribution of a $2 \mathrm{D}$ electron system for various impurity potentials characterized by $n_{i}$ and $d$. We use typically 10 different realizations for the impurity potential to calculate the average value of the physical quantities. Our calculations are carried out in a $64 \times 64$ discrete space. The size of the system is set as $L=64 a_{B}^{*}$. The average electron density is adjusted by changing the electron number $N$. We use atomic units (a. u.) to measure energy and length, i.e. $a_{B}^{*}=1$. 


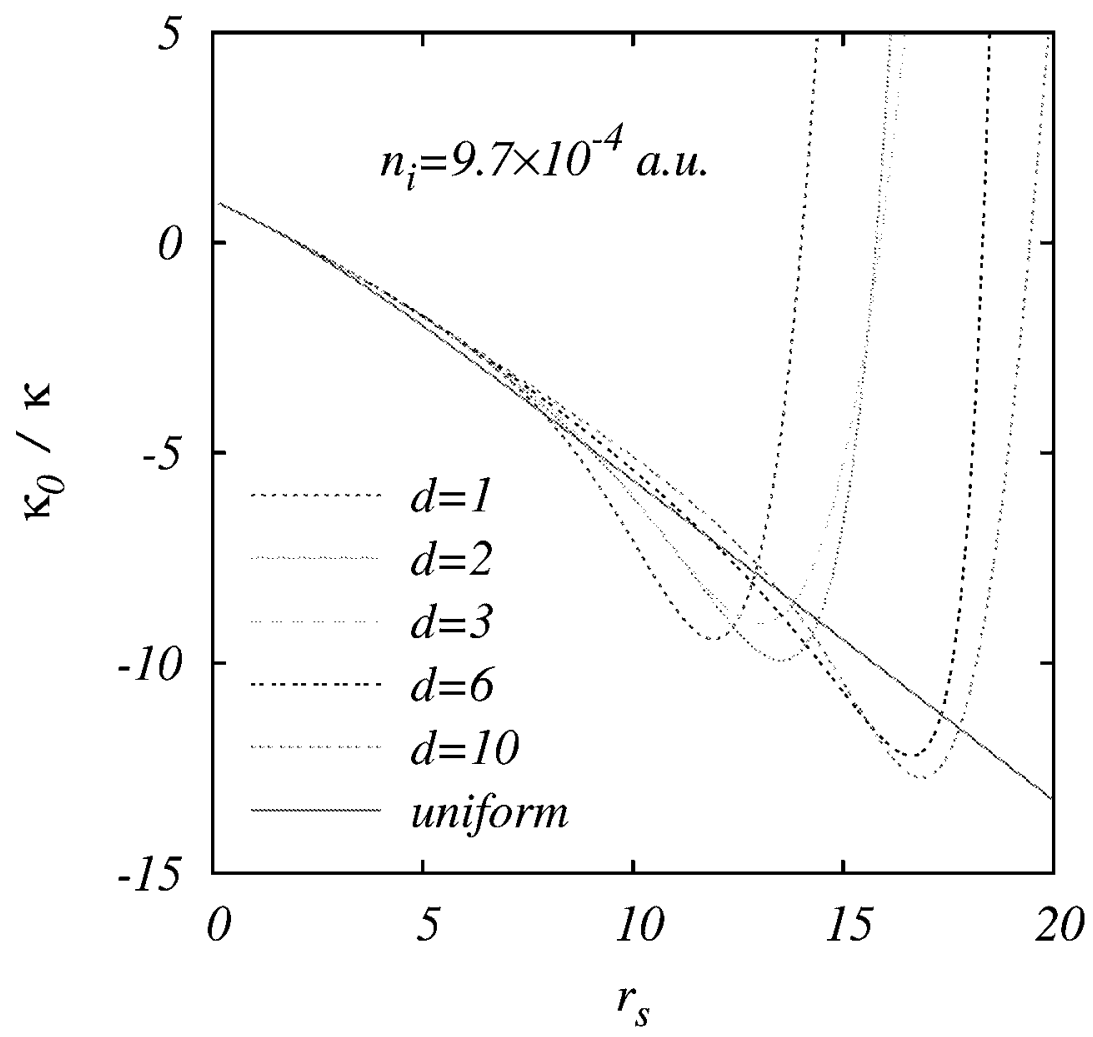

Fig. 5. Inverse compressibility scaled by its noninteracting value as a function of $r_{s}$ for a 2D electron system in the presence of charged impurities of concentration $n_{i}=0.97 \times 10^{-3}$ a.u. Distance $d$ of the impurities are given in the legend. Homogeneous system result (solid line) is the QMC calculation of Attaccalite et al. (Ref. 21)

Illustrative examples of density distributions resulting from the minimization of total energy are shown in Fig. 1 for two sets of $\left(n_{i}, d\right)$ values at various average electron densities described by $r_{s}$. We observe that electrons form high density and low density regions, an inhomogeneous distribution. Depending on the average density of the system, the high density regions may be connected to each other or form isolated patches. Approaching from the low average density side (large $r_{s}$ ), at some critical density the high density regions start to percolate through the system and form a conducting channel. This may be thought of a demonstration of metalinsulator transition in a $2 \mathrm{D}$ electron system as a percolation transition in electron density. Experiments on conductivity ${ }^{19}$ and resistance and $1 / f$ resistance noise ${ }^{24}$ have been analyzed in terms of a percolation scenario. The calculations of Fogler ${ }^{20}$ and analysis of Allison et al. ${ }^{9}$ data, on the other hand, suggest otherwise. We have not performed any systematical analysis of our calculated density distributions in view of percolation transition, thus we are not able to infer any conclusions. 


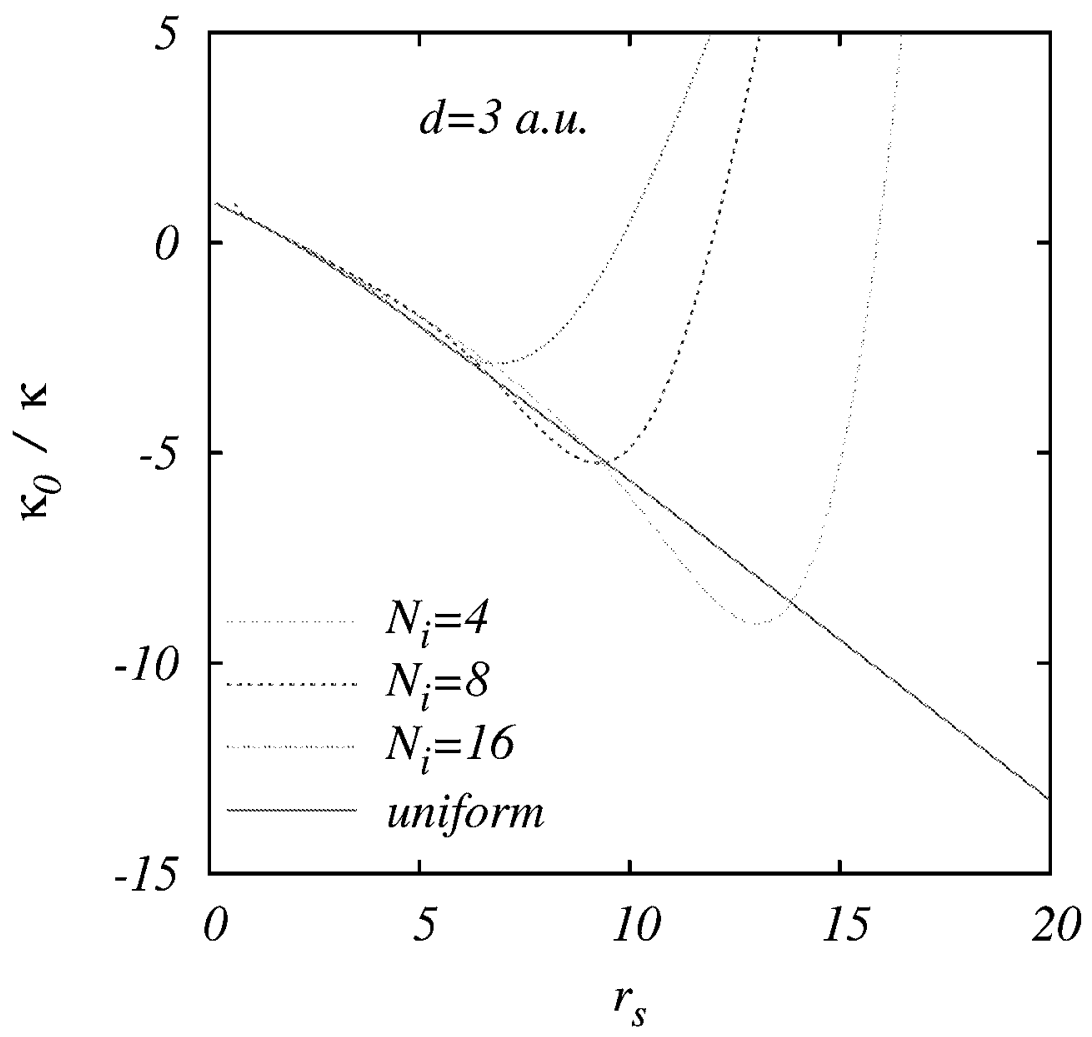

Fig. 6. Inverse compressibility scaled by its noninteracting value as a function of $r_{r} s$ for a 2D electron system in the presence of charged impurities at a setback distance $d=3 \mathrm{a}$.u. Number of impurities are given in the legend. Homogeneous system result (solid line) is the QMC calculation of Attaccalite et al. (Ref. 21)

In Fig. 2 we display the effective potential [c.f. Eq. (8)] including the Hartree, exchange-correlation, and disorder contributions. As is the density distributions we average over 10 realizations of the impurity potential for each $r_{s}$ value. It is seen that $V_{\text {eff }}$ shows the largest fluctuations when the density distribution is highest.

In Fig. 3 we show the ground state energy (per particle) as a function of $r_{s}$ for different disorder potentials. Here the impurity concentration is kept fixed at $n_{i}=0.97 \times 10^{-3} / a_{B}^{*}$ and the setback distance $d$ from the electron layer is varied. For large values of $d$ the total energy approaches to that of the homogeneous system. ${ }^{21}$ This demonstrates the reliability of our numerical approach. We also note that deviations from the clean system result manifest themselves at larger $r_{s}$ values indicating the importance of interplay between interaction and disorder effects. That is, for the same strength of impurity potential the ground-state energy is more affected at a larger $r_{s}$ value. In Fig. 4 the setback distance is fixed at $d=3$ a.u. 
and the number of charged impurities is varied. Similar qualitative effects are also observed on the ground-state energy as the disorder strength is increased.

Having calculated the ground-state energy for a $2 \mathrm{D}$ electron system in the presence of disorder, we now turn our attention to the isothermal compressibility $1 / \kappa=\left(N^{2} / A\right) \partial \mu / \partial N$ where $\mu=\partial E / \partial N$ is the chemical potential. In terms of the noninteracting system compressibility $\kappa_{0}$ we obtain

$$
\frac{\kappa_{0}}{\kappa}=-\frac{r_{s}^{3}}{4}\left[\frac{\partial E}{\partial r_{s}}-r_{s} \frac{\partial^{2} E}{\partial r_{s}^{2}}\right]
$$

where the energy $E$ is in a.u. Using the ground-state energy curves for a $2 \mathrm{D}$ disordered electron system displayed in Figs. 3 and 4, we calculate the inverse compressibility. Figures 5 and 6 show the inverse compressibility as a function of $r_{s}$ for different disorder strengths. We observe that when impurity scattering is present in the system, $\kappa_{0} / \kappa$ exhibits a minimum at some critical $r_{s}$ value, makes an upturn, becomes positive, and finally diverges at a larger $r_{s}$. This qualitative behavior seems to be quite general, in the sense that even a very weak disorder leads to it. In a fully interacting system without disorder, on the other hand, $\kappa_{0} / \kappa$ is a monotone decreasing function of $r_{s}$. The minimum of $\kappa_{0} / \kappa$ is controlled by the strength of electron-impurity interactions and through averaging by the disorder strength.

Our results for the inverse compressibility of a $2 \mathrm{D}$ electron system in the presence of disorder are in agreement with experimental findings. ${ }^{7,8,9}$ In particular, the recent experiments of Allison et al. ${ }^{9}$ performed on a variety of electron and hole systems have shown the existence of a minimum in $\kappa_{0} / \kappa$ occurring quite generally. These experiments have also measured resistivity on the same samples and showed that the metal-insulator transition (determined from $d \rho / d T=0$ ) is not related to the minimum behavior of $\kappa_{0} / \kappa$. Within our ground-state energy based calculations we cannot locate the metal-insulator transition density (or corresponding $r_{s}$ ). We expect the analysis of the solution of Kohn-Sham equations, for instance the statistical properties of single-particle energy levels may shed some light on this problem which we hope to study in future.

\section{Acknowledgments}

This work is partially supported by TUBITAK and TUBA. K. E. would like to thank TUBA for support and the hospitality of Bilkent University where part of this work was performed. We thank Dr. M. M. Fogler for discussions. We also dedicate this paper to Professor M. Tomak on the occasion of his sixtieth birthday.

\section{References}

1. E. Abrahams, S.V. Kravchenko, and M.P. Sarachik, Rev. Mod. Phys. 73, 251 (2001).

2. S.V. Kravchenko and M.P. Sarachik, Rep. Prog. Phys. 67, 1 (2004).

3. V. M. Pudalov, M. E. Gershenson, H. Kojima, N. Butch, E. M. Dizhur, G. Brunthaler, A. Prinz, and G. Bauer, Phys. Rev. Lett. 88, 196404 (2002).

4. E. Tutuc, S. Melinte, and M. Shayegan, Phys. Rev. Lett. 88, 036805 (2002). 
5. J. Zhu, H. L. Stormer, L. N. Pfeiffer, K. W. Baldwin, and K. W. West, Phys. Rev. Lett. 90, 056805 (2003).

6. W. Pan, D. C. Tsui, and B. L. Draper, Phys. Rev. B 59, 10208 (1999).

7. S. Ilani, A. Yacoby, D. Mahalu, and H. Shtrikman, Phys. Rev. Lett. 84, 3133 (2000).

8. C. Dultz and H. W. Jiang, Phys. Rev. Lett. 84, 4689 (2000).

9. G. Allison, E.A. Galaktionov, A.K. Savchenko, S.S. Safonov, M.M. Fogler, M.Y. Simmons, and D.A. Ritchie, Phys. Rev. Lett. 96, 216407 (2006).

10. O. Prus, Y. Yaish, M. Reznikov, U. Sivan, and V. Pudalov, Phys. Rev. B 67, 205407 (2003).

11. A. A. Shashkin, S. Anissimova, M. R. Sakr, S. V. Kravchenko, V. T. Dolgopolov, and T. M. Klapwijk, Phys. Rev. Lett. 96, 036403 (2006).

12. A.A. Shashkin, S. V. Kravchenko, V. T. Dolgopolov, and T. M. Klapwijk, Phys. Rev. B 66, 073303 (2002).

13. A. A. Shashkin, M. Rahimi, S. Anissimova, S. V. Kravchenko, V.T. Dolgopolov, and T. M. Klapwijk, Phys. Rev. Lett. 91, 046403 (2003); A. A. Shashkin, S. V. Kravchenko, V. T. Dolgopolov, and T. M. Klapwijk, J. Phys. A: Math. Gen. 36, 9237 (2003).

14. A. Punnoose and A.M. Finkelstein, Science 310, 289 (2005).

15. Q. M. Si and C. M. Varma, Phys. Rev. Lett. 81, 4951 (1998).

16. R. Asgari and B. Tanatar, Phys. Rev B 65, 085311 (2002).

17. J. Shi and X.C. Xie, Phys. Rev. Lett. 88, 086401 (2002).

18. B. Tanatar and D. M. Ceperley, Phys. Rev. B 39, 5005 (1989).

19. S. Das Sarma, M.P. Lilly, E.H. Hwang, L.N. Pfeiffer, K.W. West, and J.L. Reno, Phys. Rev. Lett. 94, 136401 (2005).

20. M.M. Fogler, Phys. Rev. B 69, 121409 (2004).

21. C. Attaccalite, S. Moroni, P. Gori-Giorgi, and G. B. Bachelet, Phys. Rev. Lett. 88, 256601 (2002).

22. Y. Wang, J. Wang, H. Guo, and E. Zaremba, Phys. Rev. B 52, 2738 (1995).

23. D. Heidarian and N. Trivedi, Phys. Rev. Lett. 93, 126401 (2004).

24. G. Deville, B. Leturcq, D. L'Hote, R. Tourbot, C. J. Mellor, and M. Henini, Physica E 34, 252 (2006). 